The Agriculturists 18(1): 42-55 (2020) ISSN 2304-7321 (Online), ISSN 1729-5211 (Print)

A Scientific Journal of Krishi Foundation

Indexed Journal

DOI: https://doi.org/10.3329/agric.v18i1.49458

\title{
Amelioration of Adverse Effect of Drought on Rice (Oryza sativa L.) Variety BRRI Dhan28 through Application of Poultry Litter Based Compost
}

\author{
A. K. M. M. B. Chowdhury*', M. A. $\operatorname{Hossain}^{1}$, J. Alam ${ }^{1}$, M. A. $\operatorname{Hasan}^{1}$, M. Z. Islam ${ }^{2}$ \\ ${ }^{1}$ Department of Crop Physiology and Ecology, Hajee Mohammad Danesh Science and \\ Technology University, Dinajpur, Bangladesh; ${ }^{2}$ Division of Plant Breeding, Bangladesh \\ Wheat and Maize Research Institute, Dinajpur, Bangladesh
}

*Corresponding author and Email: minarbari07@gmail.com

Received: 19 January 2020

Accepted: 25 June 2020

\begin{abstract}
An experiment was carried out at the research field of Crop Physiology and Ecology Department, Hajee Mohammad Danesh Science and Technology University, Dinajpur during January 2017 to May 2017 to evaluate the effects of poultry litter based composts on morpho-physiological, yield and yield contributing characters of BRRI dhan28, a mega rice variety of Boro season in Bangladesh, under different water stress conditions. The plants were grown in pots, and the experiment was laid out in a factorial complete randomized design (CRD) with three replications. Factor A included three levels of fertilizer and compost amendments i.e. control (recommended inorganic fertilizer), compost (10 $\left.\mathrm{t} \mathrm{ha}^{-1}\right)$ + inorganic fertilizer, compost $\left(20 \mathrm{t} \mathrm{ha}^{-1}\right)+$ without inorganic fertilizers, and factor B comprised of three water levels (continuous flooding, 100\% field capacity and 75\% field capacity). Different parameters such as plant height, tiller number, leaf number, leaf area, chlorophyll content, proline content and yield contributing characters were measured during the experiment. Most of the measured parameters were significantly influenced by irrigation levels with different fertilizer and compost amendments. The highest plant height and leaf number per hill were found in the treatment of compost $\left(10 \mathrm{t} \mathrm{ha}^{-1}\right)+$ inorganic fertilizer with $100 \%$ field capacity, though the maximum tiller number were produced by the interaction of control with flooding irrigation. The total chlorophyll was observed more in flag leaf by the interaction of control with $100 \%$ field capacity. The Proline content was observed more in flag leaf by the interaction of control with $75 \%$ field capacity. Relative water content was found more with the interaction of compost $\left(10 \mathrm{t} \mathrm{ha}^{-1}\right)+$ inorganic fertilizer with $75 \%$ field capacity. The highest effective tiller number hill ${ }^{-1}$ was found from control (19.33) at harvest. It was also observed that irrigation with 75\% field capacity produced the lowest effective tiller (15.89) hill $^{-1}$ at harvest. The longest panicle length $(23.12 \mathrm{~cm})$ was observed with the combined effect of compost (10 t $\left.\mathrm{ha}^{-1}\right)+$ inorganic with $75 \%$ field capacity. Thousand grain weight was found highest $(18.83 \mathrm{~g})$ in the interaction of compost $\left(20 \mathrm{t} \mathrm{ha}^{-1}+\right.$ without inorganic) with $75 \%$ field capacity. The interaction of compost $\left(20 \mathrm{t} \mathrm{ha}^{-1}+\right.$ without inorganic) and flooding irrigation gave the highest grain yield $(33.17 \mathrm{~g}$ pot $\left.^{-1}\right)$, which was statistically similar to the treatment of compost $\left(10 \mathrm{t} \mathrm{ha}^{-1}+\right.$ inorganic) with flooding irrigation. The highest harvest index was found from compost $\left(20 \mathrm{tha}^{-1}+\right.$ without inorganic) with flooding irrigation.
\end{abstract}

Keywords: Rice, Fertilizer, Poultry litter based compost, drought, irrigation, yield 


\section{Introduction}

Rice (Oryza sativa L.) is the principal food crop in Bangladesh and about $80 \%$ of the total arable lands are used for rice (aus, aman and boro) cultivation (BBS, 2014). It is the staple food for nearly half of the world's population as well as for 148.10 million people of Bangladesh (AIS, 2008). In Asia, where approximately $90 \%$ of world's rice is produced and consumed. Rice plays absolutely dominant role in Bangladesh agriculture as it covers 77.96 percent of total cropped area (AIS, 2008). Rice-rice cropping system is the most important cropping system in Bangladesh. Continuous cultivation of this highly exhaustive cropping sequence in most of the irrigated fertile lands has resulted in the decline of soil physio-chemical condition in general and particularly soil organic matter (SOM) content due to less addition of organic matter and higher microbial activity (Adugna, 2016; Liu et al., 2013).

In general, the low yield of boro rice is due to several factors. Sources of $\mathrm{N}$ fertilizer are an important factor for obtaining higher yields. It is well known that inorganic fertilizers supply only nutrients in soil but organic manure supplies nutrients and at the same time improves soil quality. The long term impact of chemical fertilizers on soils and environment is harmful. Use of imbalanced nutrients in the soils may be harmful in the long run causing soils an unproductive one. It is true that sustainable production of crops cannot be maintained by using only chemical fertilizers and similarly it is not possible to obtain higher crop yield by using organic manure alone (Bair, 1990). Proper identification and management of soil fertility problems are prerequisite for boosting crop production and sustaining higher yields over a long period of time. -A judicious combination of organic and inorganic sources of nutrients is necessary for sustainable agriculture that can ensure food production with high quality (Nambiar, 1991). Integrated use of organic manure and chemical fertilizers would be quite promising not only in providing greater stability in production, but also in maintaining better soil fertility. The two year research at BRRI revealed that the application of poultry manure@ $3 \mathrm{t} \mathrm{ha}^{-}$ ${ }^{1}$ year $^{-1}$ improved rice productivity as well as prevented the soil resources from degradation (Rahman et al., 2018). Poultry manure is another good source of nutrients in soil. Meelu and Singh (1991) showed that $4 \mathrm{t} \mathrm{ha}^{-1}$ poultry manure along with $60 \mathrm{~kg} \mathrm{~N} \mathrm{ha}^{-1}$ as urea produced grain yield of crop similar to that with $120 \mathrm{~kg} \mathrm{Nha}^{-1}$ as urea alone. But direct application of poultry manure have negative effect on soil health (Urra et al., 2019). That's why it needs be composted. Organic compost can supply a good amount of plant nutrients and therefore can contribute to crop yields (Chowdhury et al., 2013; Najafi, and Abbasi. 2009).

Therefore, it is necessary to use fertilizer and compost in an integrated way in order to obtain sustainable crop yield without declining soil fertility. The increasing land use intensity has resulted in a great exhaustion of nutrient in soils. In Bangladesh, most of the cultivated soils have less than $1.5 \%$ organic matter while a good agricultural soil should contain at least $2 \%$ organic matter.

Moreover, this important component of soil is declining with time due to intensive cropping and use of higher dose chemical fertilizers. Whereas, soil organic matter improves the physicochemical properties of the soil and ultimately promotes crop production. The application of organic manure decreases the need of irrigation water (Marinari et al., 2000). The application of different fertilizers and organic compost influences the physical and chemical properties of soil and enhances the biological activities. It is also positively correlated with soil porosity and enzymatic activity (Reardon and Wuest, 2016, Liu et al., 2013 and Lin et al., 2011). Applications of both chemical and organic fertilizers need to be applied for the improvement of soil physical properties and supply of essential plant nutrients for higher yield (Diacono et al., 2010). 
Boro rice is grown under irrigated condition during November to May. There is almost no rainfall during this period. Some sporadic rainfall may occur at late April and May with thunderstorms (Shelly et al., 2016). That's why irrigation is considered as one of the most important factors to cultivate boro rice. BRRI dhan28 is a high yielding mega rice variety of boro season produced by BRRI.

The present investigation was therefore, undertaken to develop a suitable integrated dose of poultry litter based organic compost and inorganic fertilizer in combination of different levels of water to find out the interaction effects on yield as well as yield components of BRRI dhan28.

\section{Materials and Methods}

The experiment was conducted at the Crop Physiology and Ecology Research Field, Hajee Mohammad Danesh Science and Technology University (HSTU), Dinajpur during January to May 2017. The experiment was laid out in a factorial complete randomized design (CRD) with three replications. Factor A included three (3) fertilizer levels i.e. control (inorganic), compost $\left(10 \mathrm{t} \mathrm{ha}^{-1}\right)+$ inorganic, compost $(20 \mathrm{t} \mathrm{ha}$ $\left.{ }^{1}\right)+$ without inorganic fertilizers and factor $\mathrm{B}$ comprised of three water levels (continuous flooding, $100 \%$ field capacity and $75 \%$ field capacity). A 15 litter bucket was used as a pot. Each pot was filled with $15 \mathrm{~kg}$ soil. Above mentioned rates of compost and fertilizer dose (recommended) were given in each pot before transplanting of rice. Compost was prepared from poultry manure with different bulking agents (Kobra, 2016). Field capacity was measured through gravimetric method before use. Watering was done according to treatments. Each pot contained two seedlings of 25 days old. Weeding and other intercultural operations were done as per requirement.

\subsection{Data collection and analysis}

Plants from each pot were randomly selected at 30 days, vegetative (maximum tillering), booting, anthesis and maturity stage after transplanting for collecting data on plant height, number of leaves, leaf area, stem dry weight, leaf dry weight and root dry weight. Plant height was measured from base to the tip of the top leaf (at 30 DAT, vegetative \& reproductive stage) and tip of the top panicle (for maturity stage). Leaf area of all the green leaves was measured by leaf area meter (CI-202 AREA METER). At each sampling, plants were uprooted and separated into leaf, stem and root. After separating the different parts of the plants, individual component were oven dried at $70^{\circ} \mathrm{C}$ for at least 72 hours and weighed.

Chlorophyll content of the flag leaf at anthesis stage was estimated according to Witham et al. (1986). $0.25 \mathrm{~g}$ of leaf was taken from middle position of the flag leaf in a brown bottle containing $25 \mathrm{ml}$ of $80 \%$ aqueous acetone. The bottles were kept in dark for 48 hours. The optical density of this green colored extract was determined against $80 \%$ acetone as blank using spectrophotometer (SPECTRO UV-VIS RS Spectrophotometer, Labo Med, Inc.) at 645 and $663 \mathrm{~nm}$. Chlorophyll-a, chlorophyll-b and total chlorophyll of fresh leaf was determined using following formula-

Ch-a $(\mathrm{mg} / \mathrm{g})=\left[12.7\left(\mathrm{D}_{663}\right)-2.69\left(\mathrm{D}_{645}\right)\right] * \mathrm{~V} / 1000 \mathrm{~W}$

Ch-b $(\mathrm{mg} / \mathrm{g})=\left[22.9\left(\mathrm{D}_{645}\right)-4.68\left(\mathrm{D}_{663}\right)\right] * \mathrm{~V} / 1000 \mathrm{~W}$

Total $(\mathrm{mg} / \mathrm{g})=\left[20.21\left(\mathrm{D}_{645}\right)+8.02\left(\mathrm{D}_{663}\right)\right] * \mathrm{~V} / 1000 \mathrm{~W}$

Where, $\mathrm{D}_{645} \& \mathrm{D}_{663}=$ spectrophotometer reading at $645 \mathrm{~nm} \& 663 \mathrm{~nm}, \mathrm{~V}=25 \mathrm{ml}$ of $80 \%$ acetone each bottle (Volume of solution), $\mathrm{W}=0.25 \mathrm{~g}$ leaf each bottle (Fresh weight of leaf sample).

Relative leaf water content was determined from the flag leaves during anthesis stage of rice crop. The leaves were collected at 8.00 am. 12.00 noon and $4 \mathrm{pm}$. Three leaves were taken from each replication. There fresh weights of the leaf samples were taken immediately and then were sinked into water and kept in petridish for four hours. After four hours when the cells of the leaves become fully turgid, they were taken out from water and their turgid weights were taken 
immediately removing adhere surface water with blotting paper by an electric balance. Then the leaves were dried in an oven and weighted. The relative leaf water content was calculated from the following formula (Barrs and Weatherly, 1962).

$$
\begin{aligned}
& \text { Relative leaf water content }(\%)= \\
& \text { Fresh weight - Dry weight } \\
& \text { Turgid weight - Dry weight }
\end{aligned}
$$

At maturity, harvesting was started at 90 days after transplanting and ended at 95 days after transplanting due to different date of maturity. Grains obtained from unit pot were sun dried and weighed carefully. The harvested crop was threshed pot-wise (g/pot). And 1000- seed weight were also recorded from randomly selected plants of each pot.

The data were analyzed by partitioning the total variance using STATA 12 program and the treatment means were compared using Tukey's Test.

\section{Results and Discussion}

\subsection{Plant height}

Plant height was significantly affected by interaction between fertilizer and different water level as presented in Table 1. Results indicated that the tallest plants $(44.8,84.6,85.03,88.2$ and $89.13 \mathrm{~cm}$ at 30 DAT, vegetative, booting, anthesis and maturity stages, respectively) were found in $\mathrm{F}_{2}$ (compost-10 $\mathrm{t} \mathrm{ha}^{-1}+$ inorganic) fertilizer with $100 \%$ field capacity water level, which was significantly different from other treatments. The shortest plants (37.9,72.63,78.1,77.6 and $81.77 \mathrm{~cm}$ at 30 DAT, vegetative, booting, anthesis and maturity stages, respectively) were found from compost $\left(20 \mathrm{tha}^{-1}\right.$ + without inorganic) fertilizer with $75 \%$ field capacity water level. Nutrient availability may occur more at $\mathrm{F}_{2 \times} \mathrm{I}_{2}$ interaction that's why it gave higher plant height. Abro et al. (2002) and Hossain (2008) also reported the similar result that fertilizer and irrigation enhanced plant height in rice.

\subsection{Number of leaves hill $^{-1}$}

Leaf number hill ${ }^{-1}$ was significantly affected by interaction between poultry based compost and different water levels as presented in Table 2. Results indicated that the highest leaf number hill $^{-1}$ at vegetative (62.67) and booting (55.67) stages was obtained from the treatment $\mathrm{F}_{1 \times} \mathrm{I}_{1}$ (inorganic with flooding irrigation), whereas $\mathrm{F}_{1 \times} \mathrm{I}_{2}$ (inorganic with $100 \%$ field capacity) gave the highest result at 30 DAT and anthesis, which was significantly different from all other treatments. The lowest leaf number hill ${ }^{-1}$ (47 and 53.67 at vegetative stages respectively) was found from the treatment $\mathrm{F}_{3 \times} \mathrm{I}_{3}$ (compost-20 $\mathrm{tha}^{-1}$ + without inorganic with $75 \%$ field capacity). At booting and anthesis stages the treatment $\mathrm{F}_{1 \times} \mathrm{I}_{3}$ (inorganic with $75 \%$ field capacity) gave the lowest leaves hill $^{-1}$ (45.67 and 80). Water may play an important role for the nutrient availability which may help to increase number of leaf as reported by Akter (2011).

\subsection{Number of tiller hill $^{-1}$}

Tiller number hill ${ }^{-1}$ was significantly affected by interaction between fertilizer and different water level (Table 3). Results indicated that the highest tiller number hill $^{-1}(17,12.67,15.33$, 23.33, and 25 at vegetative, booting, anthesis and maturity stages, respectively) were found in $\mathrm{F}_{1 \times} \mathrm{I}_{1}$ (inorganic fertilizer with flooding irrigation), which was significantly different from all other treatments and the lowest tiller number hill ${ }^{-1}(11$, 10 and 8 at vegetative, booting stages respectively) was found from treatment $\mathrm{F}_{3 \times} \mathrm{I}_{3}$ (compost-20 t ha ${ }^{-1}+$ without inorganic with $75 \%$ field capacity) and also at anthesis and maturity the lowest tiller number hill ${ }^{-1}$ (15.67 and 17, respectively was found from $\mathrm{F}_{1 \times} \mathrm{I}_{3}$ (inorganic with $75 \%$ field capacity). Nitrogen availability may play important role for tiller generation. Inorganic fertilizer is good source of available nitrogen with flooding irrigation as reported by Islam et al. (2014), Shaha (2014) and Hossain (2010). 
Table1. Effect of fertilizer and water level on plant height $(\mathrm{cm})$ of rice (BRRI dhan28) at different days after transplanting.

\begin{tabular}{|c|c|c|c|c|c|c|}
\hline Fertilizer & Irrigation & $30 \mathrm{DAT}$ & Vegetative & Booting & Anthesis & Harvest \\
\hline \multirow[t]{3}{*}{$\mathrm{F}_{1}$} & $\mathrm{I}_{1}$ & $40.33 b$ & $76.33 b$ & $84.57 \mathrm{a}$ & $83.2 \mathrm{~b}$ & $87.6 \mathrm{a}$ \\
\hline & $\mathrm{I}_{2}$ & $40 \mathrm{~b}$ & $75.37 \mathrm{bc}$ & $78.47 \mathrm{~b}$ & $77.6 \mathrm{c}$ & $88.4 \mathrm{a}$ \\
\hline & $\mathrm{I}_{3}$ & $41.33 b c$ & $76 \mathrm{~b}$ & $78.43 b$ & $87.57 \mathrm{a}$ & $87.73 a$ \\
\hline \multirow[t]{3}{*}{$\mathrm{F}_{2}$} & $\mathrm{I}_{1}$ & $41.93 \mathrm{bc}$ & $83.67 \mathrm{a}$ & $83.6 \mathrm{a}$ & $83.53 b$ & $83.43 b$ \\
\hline & $\mathrm{I}_{2}$ & $44.8 \mathrm{a}$ & $84.6 \mathrm{a}$ & $85.03 \mathrm{a}$ & $88.2 \mathrm{a}$ & $89.13 \mathrm{a}$ \\
\hline & $\mathrm{I}_{3}$ & $40.77 \mathrm{~b}$ & $74 \mathrm{c}$ & $80.13 \mathrm{ab}$ & $84.53 \mathrm{ab}$ & $82.13 \mathrm{ab}$ \\
\hline \multirow[t]{3}{*}{$\mathrm{F}_{3}$} & $\mathrm{I}_{1}$ & $39.3 \mathrm{bc}$ & $81.33 \mathrm{a}$ & $78.13 b$ & $82.17 \mathrm{ab}$ & $82.87 \mathrm{~b}$ \\
\hline & $\mathrm{I}_{2}$ & $41.1 \mathrm{bc}$ & $71.27 \mathrm{~cd}$ & $82.1 \mathrm{a}$ & $77.97 \mathrm{c}$ & $84.07 \mathrm{ab}$ \\
\hline & $\mathrm{I}_{3}$ & $37.9 \mathrm{c}$ & $72.63 \mathrm{~cd}$ & $78.1 \mathrm{~b}$ & $77.6 \mathrm{c}$ & $81.77 \mathrm{c}$ \\
\hline \multicolumn{2}{|c|}{ Level of significance } & $*$ & $*$ & $* *$ & $*$ & $*$ \\
\hline \multicolumn{2}{|c|}{$\mathrm{CV}(\%)$} & 4.56 & 6.23 & 7.13 & 6.27 & 7.81 \\
\hline
\end{tabular}

In a column, means followed by different letter(s) differed significantly by Tukey's test at $\mathrm{P} \leq 5 \%$ level of probability. $\mathrm{F}_{1}$ - Inorganic (control), $\mathrm{F}_{2}-$ Compost $\left(10 \mathrm{t} \mathrm{ha}{ }^{-1}\right)+$ inorganic, $\mathrm{F}_{3}-$ Compost $(20 \mathrm{tha}$ $\left.{ }^{1}\right)+$ without inorganic and $\mathrm{I}_{1}$ - continuous flooding, $\mathrm{I}_{2}-100 \%$ field capacity, $\mathrm{I}_{3}-75 \%$ field capacity.

Table 2. Effect of fertilizer and water level on leaf number of rice (BRRI dhan28) at different days after transplanting (DAT).

\begin{tabular}{cccccc}
\hline Fertilizer & Irrigation & $30 \mathrm{DAT}$ & Vegetative & Booting & Anthesis \\
\hline $\mathrm{F}_{1}$ & $\mathrm{I}_{1}$ & $50.67 \mathrm{bc}$ & $62.67 \mathrm{a}$ & $55.67 \mathrm{a}$ & $91 \mathrm{~b}$ \\
& $\mathrm{I}_{2}$ & $65.67 \mathrm{a}$ & $56.67 \mathrm{c}$ & $50.67 \mathrm{~b}$ & $106.33 \mathrm{a}$ \\
& $\mathrm{I}_{3}$ & $56 \mathrm{~b}$ & $54.33 \mathrm{~cd}$ & $45.67 \mathrm{~d}$ & $80 \mathrm{c}$ \\
$\mathrm{F}_{2}$ & $\mathrm{I}_{1}$ & $60 \mathrm{a}$ & $58.67 \mathrm{ab}$ & $54.33 \mathrm{ab}$ & $95.33 \mathrm{ab}$ \\
& $\mathrm{I}_{2}$ & $67.33 \mathrm{a}$ & $62.33 \mathrm{a}$ & $49.67 \mathrm{bc}$ & $91.33 \mathrm{~b}$ \\
& $\mathrm{I}_{3}$ & $48 \mathrm{~d}$ & $59.67 \mathrm{ab}$ & $52 \mathrm{~b}$ & $90.87 \mathrm{~b}$ \\
$\mathrm{~F}_{3}$ & $\mathrm{I}_{1}$ & $54.33 \mathrm{bc}$ & $57 \mathrm{c}$ & $55 \mathrm{a}$ & $89 \mathrm{~b}$ \\
& $\mathrm{I}_{2}$ & $63.67 \mathrm{a}$ & $47 \mathrm{~d}$ & $55 \mathrm{a}$ & $78 \mathrm{c}$ \\
& $\mathrm{I}_{3}$ & $47 \mathrm{~d}$ & $53.67 \mathrm{~cd}$ & $44.67 \mathrm{c}$ & $89 \mathrm{~b}$ \\
\hline \multicolumn{2}{c}{ Level of significance } & $*$ & $* *$ & $*$ & $* *$ \\
\multicolumn{2}{c}{$\mathrm{CV}(\%)$} & 6.26 & 7.34 & 4.12 & 6.27 \\
\hline
\end{tabular}

In a column, means followed by different letter(s) differed significantly by Tukey's test at $\mathrm{P} \leq 5 \%$ level of probability. $\mathrm{F}_{1}$ - Inorganic (control), $\mathrm{F}_{2}$ - Compost $\left(10 \mathrm{t} \mathrm{ha}{ }^{-1}\right)+$ inorganic, $\mathrm{F}_{3}$ - Compost $\left(20 \mathrm{t} \mathrm{ha}{ }^{-1}\right)$ + without inorganic and $\mathrm{I}_{1}$ - continuous flooding, $\mathrm{I}_{2}-100 \%$ field capacity, $\mathrm{I}_{3}-75 \%$ field capacity.

\subsection{Number of effective tillers hill ${ }^{-1}$}

Number of effective tillers hill ${ }^{-1}$ was significantly affected by interaction between fertilizer and different levels of irrigation (Figure. 1). Results indicated that the highest number of effective tillers hill ${ }^{-1}(19.33,19)$ was found in the combination of $\mathrm{F}_{1 \times} \mathrm{I}_{1}$ (inorganic fertilizer with flooding). The lowest number of effective tillers hill $^{-1}(15.33,9.33)$ was found from the treatment combination of $\mathrm{F}_{3 \times} \mathrm{I}_{3}$ (20ton/ ha with $75 \%$ FC) and the finding was at par with that of Hoshain (2010) and Fakhrul (2013). 
Table 3. Effect of fertilizer and irrigation level on tiller number of rice (BRRI dhan28) at different days after transplanting (DAT).

\begin{tabular}{ccccccc}
\hline Fertilizer & Irrigation & $30 \mathrm{DAT}$ & Vegetative & Booting & Anthesis & Maturity \\
\hline $\mathrm{F}_{1}$ & $\mathrm{I}_{1}$ & $17 \mathrm{a}$ & $12.67 \mathrm{a}$ & $15.33 \mathrm{a}$ & $23.33 \mathrm{a}$ & $25 \mathrm{a}$ \\
& $\mathrm{I}_{2}$ & $14 \mathrm{bc}$ & $11.67 \mathrm{~b}$ & $11.67 \mathrm{ab}$ & $24.67 \mathrm{a}$ & $23 \mathrm{a}$ \\
& $\mathrm{I}_{3}$ & $13.67 \mathrm{bc}$ & $13 \mathrm{a}$ & $8 \mathrm{c}$ & $15.67 \mathrm{c}$ & $17 \mathrm{c}$ \\
$\mathrm{F}_{2}$ & $\mathrm{I}_{1}$ & $13.67 \mathrm{bc}$ & $12.33 \mathrm{a}$ & $13.67 \mathrm{a}$ & $22.67 \mathrm{ab}$ & $23.33 \mathrm{a}$ \\
& $\mathrm{I}_{2}$ & $15.67 \mathrm{a}$ & $13.33 \mathrm{a}$ & $11.33 \mathrm{ab}$ & $20 \mathrm{~b}$ & $20 \mathrm{~b}$ \\
& $\mathrm{I}_{3}$ & $11.33 \mathrm{c}$ & $14.67 \mathrm{a}$ & $7.67 \mathrm{bc}$ & $15.67 \mathrm{c}$ & $18.33 \mathrm{bc}$ \\
$\mathrm{F}_{3}$ & $\mathrm{I}_{1}$ & $12 \mathrm{c}$ & $12.67 \mathrm{a}$ & $12.33 \mathrm{ab}$ & $23 \mathrm{ab}$ & $23.33 \mathrm{a}$ \\
\multicolumn{2}{c}{ Level of significance } & $17.67 \mathrm{a}$ & $10.33 \mathrm{c}$ & $12.33 \mathrm{ab}$ & $19.67 \mathrm{bc}$ & $23.33 \mathrm{a}$ \\
\multicolumn{2}{c}{$\mathrm{CV}(\%)$} & $11 \mathrm{~cd}$ & $10 \mathrm{c}$ & $8 \mathrm{c}$ & $20.33 \mathrm{~b}$ & $18.33 \mathrm{bc}$ \\
\hline
\end{tabular}

In a column, means followed by different letter(s) differed significantly by Tukey's test at $\mathrm{P} \leq 5 \%$ level of probability. $\mathrm{F}_{1}$ - Inorganic (control), $\mathrm{F}_{2^{-}}$Compost $\left(10 \mathrm{t} \mathrm{ha}^{-1}\right)+$ inorganic, $\mathrm{F}_{3}-$ Compost $\left(20 \mathrm{t} \mathrm{ha}^{-}\right.$ $\left.{ }^{1}\right)+$ without inorganic and $\mathrm{I}_{1}$ - continuous flooding, $\mathrm{I}_{2}-100 \%$ field capacity, $\mathrm{I}_{3}-75 \%$ field capacity.

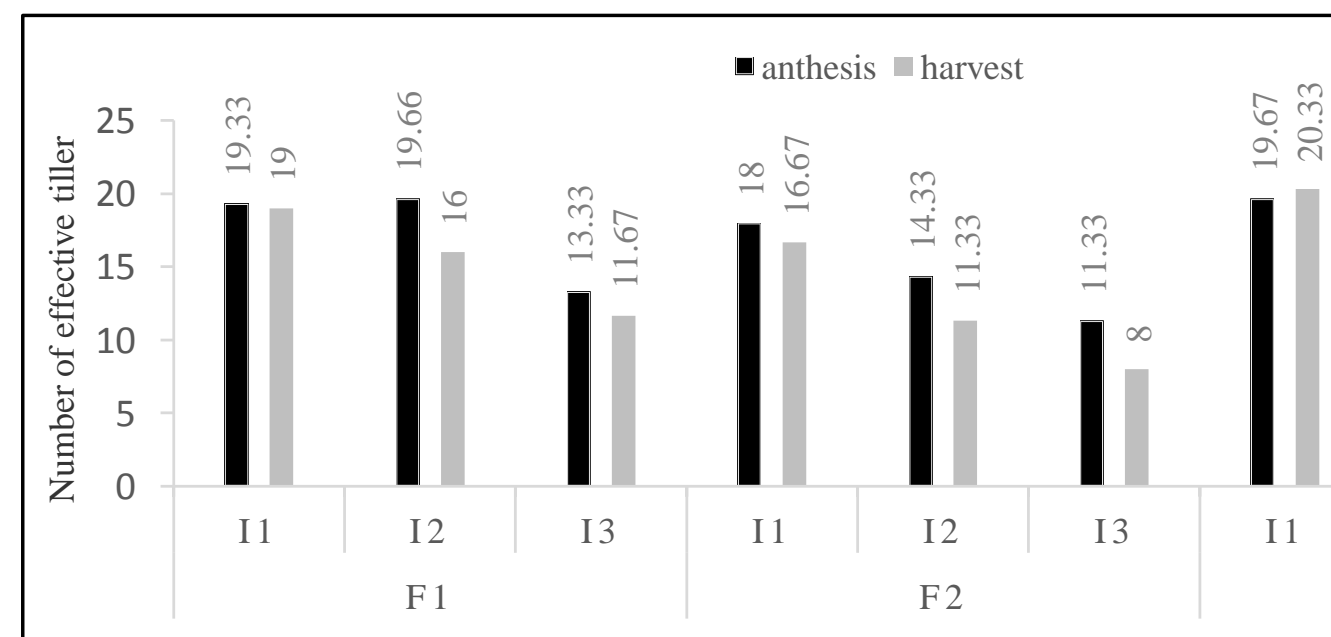

$\mathrm{F}_{1}$ - Inorganic (control), $\mathrm{F}_{2}-$ Compost $\left(10 \mathrm{t} \mathrm{ha}^{-1}\right)+$ inorganic, $\widetilde{\mathrm{F}}_{3}-$ Compost $\left(20 \mathrm{t} \mathrm{ha}^{-1}\right)+$ without inorganic and $\mathrm{I}_{1}$ - continuous flooding, $\mathrm{I}_{2}-100 \%$ field capacity, $\mathrm{I}_{3}-75 \%$ field capacity.

\subsection{Stem dry weight per plant}

Stem dry weight per plant was affected by interaction between fertilizer and different water level as presented in Table 4. At vegetative stage, the highest stem dry weight per plant $\left(9.07 \mathrm{~g}\right.$ ) was obtained in $\mathrm{F}_{1 \times} \mathrm{I}_{3}$ (inorganic with $75 \%$ field capacity) and the lowest stem dry weight per plant $(5.87 \mathrm{~g})$ was obtained in $\mathrm{F}_{2 \times} \mathrm{I}_{2}$ (compost $10 \mathrm{t} \mathrm{ha}^{-1}+$ inorganic with $\mathrm{I}_{2} 100 \%$ field capacity).

At booting stage, the highest stem dry weight per plant $\left(19.18 \mathrm{~g}\right.$ ) was obtained in $\mathrm{F}_{1 \times} \mathrm{I}_{1}$ (inorganic with flooding) and the lowest stem dry weight per plant $(11.58 \mathrm{~g})$ was obtained in $\mathrm{F}_{3 \times} \mathrm{I}_{3}$ (compost-20t ha ${ }^{-1}+$ without inorganic with $75 \%$ 
field capacity). Fakhrul (2013) found the similar result that inorganic fertilizer and irrigation increased stem dry weight per plant.

At anthesis stage, the highest stem dry weight per plant $(65.83 \mathrm{~g})$ was obtained in $\mathrm{F}_{1 \times} \mathrm{I}_{1}$ (inorganic with flooding) which was statistically similar to $\mathrm{F}_{3 \times} \mathrm{I}_{3}$ (compost-20 $\mathrm{t} \mathrm{ha}^{-1}+$ without inorganic with $75 \%$ Field capacity) (60.16g). The lowest stem dry weight per plant $(28.52 \mathrm{~g})$ was recorded from $\mathrm{F}_{2 \times} \mathrm{I}_{2}$ (compost ${ }^{-1} 0 \mathrm{t} \mathrm{ha}^{-1}+$ inorganic with $100 \%$ field capacity).

Table 4. Effect of fertilizer and water level on stem dry weight (g) of rice (BRRI dhan28) at different days after transplanting.

\begin{tabular}{|c|c|c|c|c|}
\hline Fertilizer & Irrigation & Vegetative & Booting & Anthesis \\
\hline \multirow[t]{3}{*}{$\mathrm{F}_{1}$} & $\mathrm{I}_{1}$ & $6.84 \mathrm{bc}$ & $19.18 \mathrm{a}$ & $65.83 \mathrm{a}$ \\
\hline & $\mathrm{I}_{2}$ & $6.34 \mathrm{bc}$ & $12.77 \mathrm{c}$ & $37.88 \mathrm{c}$ \\
\hline & $\mathrm{I}_{3}$ & $9.07 \mathrm{a}$ & $12.91 \mathrm{c}$ & $39.81 b c$ \\
\hline \multirow[t]{3}{*}{$\mathrm{F}_{2}$} & $\mathrm{I}_{1}$ & $7.3 b$ & $17.41 \mathrm{~b}$ & $55.03 \mathrm{~b}$ \\
\hline & $\mathrm{I}_{2}$ & $5.87 \mathrm{~d}$ & $15.15 b$ & $28.52 \mathrm{~d}$ \\
\hline & $\mathrm{I}_{3}$ & $6.80 \mathrm{bc}$ & $13.55 \mathrm{bc}$ & $37.50 \mathrm{c}$ \\
\hline \multirow[t]{3}{*}{$\mathrm{F}_{3}$} & $\mathrm{I}_{1}$ & $7.62 b$ & $17.75 b$ & $57.75 b$ \\
\hline & $\mathrm{I}_{2}$ & $5.76 \mathrm{c}$ & $17.25 b$ & $32.30 \mathrm{~d}$ \\
\hline & $\mathrm{I}_{3}$ & $7.70 \mathrm{~b}$ & $11.58 \mathrm{~d}$ & $60.16 \mathrm{ab}$ \\
\hline \multicolumn{2}{|c|}{ Level of significance } & $*$ & $*$ & $*$ \\
\hline \multicolumn{2}{|c|}{$\mathrm{CV}(\%)$} & 6.78 & 4.68 & 5.81 \\
\hline
\end{tabular}

In a column, means followed by different letter(s) differed significantly by Tukey's test at $\mathrm{P} \leq 5 \%$ level of probability. $\mathrm{F}_{1}$ - Inorganic (control), $\mathrm{F}_{2}$ - Compost $\left(10 \mathrm{tha}^{-1}\right)+$ inorganic, $\mathrm{F}_{3}-$ Compost $(20 \mathrm{t}$ ha $\left.{ }^{1}\right)+$ without inorganic and $\mathrm{I}_{1}$ - continuous flooding, $\mathrm{I}_{2}-100 \%$ field capacity, $\mathrm{I}_{3}-75 \%$ field capacity.

Table 5. Effect of fertilizer and water level on leaf dry weight $(\mathrm{g})$ of rice (BRRI dhan28) at different days after transplanting.

\begin{tabular}{|c|c|c|c|c|}
\hline Fertilizer & Irrigation & Vegetative & Booting & Anthesis \\
\hline \multirow[t]{3}{*}{$\mathrm{F}_{1}$} & $\mathrm{I}_{1}$ & $6.44 \mathrm{~b}$ & $8.11 \mathrm{~b}$ & $11.34 \mathrm{~b}$ \\
\hline & $\mathrm{I}_{2}$ & $5.77 b$ & $5.92 b c$ & $11.31 \mathrm{~b}$ \\
\hline & $\mathrm{I}_{3}$ & $5.97 \mathrm{~b}$ & $4.76 c$ & $11.08 \mathrm{~b}$ \\
\hline \multirow[t]{3}{*}{$\mathrm{F}_{2}$} & $\mathrm{I}_{1}$ & $7.36 \mathrm{a}$ & $9.65 \mathrm{a}$ & $15.39 \mathrm{a}$ \\
\hline & $\mathrm{I}_{2}$ & $5.52 b$ & $7.87 b$ & $11.25 \mathrm{~b}$ \\
\hline & $\mathrm{I}_{3}$ & $4.05 \mathrm{c}$ & $4.6 \mathrm{~d}$ & $10.33 \mathrm{bc}$ \\
\hline \multirow[t]{3}{*}{$\mathrm{F}_{3}$} & $\mathrm{I}_{1}$ & $7.35 \mathrm{a}$ & $9.85 \mathrm{a}$ & $16.43 a$ \\
\hline & $\mathrm{I}_{2}$ & $4.37 \mathrm{c}$ & $7.98 b$ & $9.59 \mathrm{c}$ \\
\hline & $\mathrm{I}_{3}$ & $7.57 \mathrm{a}$ & $6.04 \mathrm{bc}$ & $11.6 \mathrm{~b}$ \\
\hline \multicolumn{2}{|c|}{ Level of significance } & $*$ & $*$ & $*$ \\
\hline \multicolumn{2}{|c|}{$\mathrm{CV}(\%)$} & 7.68 & 5.16 & 6.31 \\
\hline
\end{tabular}

In a column, means followed by different letter(s) differed significantly by Tukey's test at $\mathrm{P} \leq 5 \%$ level of probability. $\mathrm{F}_{1}$ - Inorganic (control), $\mathrm{F}_{2^{-}}$Compost $\left(10 \mathrm{t} \mathrm{ha}^{-1}\right)+$ inorganic, $\mathrm{F}_{3}-$ Compost $\left(20 \mathrm{t} \mathrm{ha}^{-}\right.$ $\left.{ }^{1}\right)+$ without inorganic and $\mathrm{I}_{1}$ - continuous flooding, $\mathrm{I}_{2}-100 \%$ field capacity, $\mathrm{I}_{3}-75 \%$ field capacity. 
Table 6. Effect of fertilizer and irrigation on root dry weight $(\mathrm{g})$ of rice (BRRI dhan28) at different days after transplanting.

\begin{tabular}{|c|c|c|c|c|}
\hline Fertilizer & Irrigation & Vegetative & Booting & Anthesis \\
\hline \multirow{3}{*}{$\mathrm{F}_{1}$} & $\mathrm{I}_{1}$ & $15.05 \mathrm{ab}$ & $34.9 \mathrm{a}$ & $54.62 \mathrm{a}$ \\
\hline & $\mathrm{I}_{2}$ & $8.11 b c$ & $21.74 \mathrm{~b}$ & $23.38 \mathrm{bc}$ \\
\hline & $\mathrm{I}_{3}$ & $8.89 b c$ & $33.27 \mathrm{a}$ & $19.95 \mathrm{c}$ \\
\hline \multirow{3}{*}{$\mathrm{F}_{2}$} & $\mathrm{I}_{1}$ & $12.19 \mathrm{~b}$ & $13.04 b c$ & $37.26 \mathrm{~b}$ \\
\hline & $\mathrm{I}_{2}$ & $8.75 b c$ & $13.54 b c$ & $24.27 b c$ \\
\hline & $\mathrm{I}_{3}$ & $6.72 \mathrm{~d}$ & $21.58 \mathrm{~b}$ & $14.76 \mathrm{~d}$ \\
\hline \multirow[t]{3}{*}{$\mathrm{F}_{3}$} & $\mathrm{I}_{1}$ & $18.6 \mathrm{a}$ & $11.34 \mathrm{c}$ & $22.29 b c$ \\
\hline & $\mathrm{I}_{2}$ & $7.90 \mathrm{~d}$ & $9.13 c$ & $15.14 \mathrm{c}$ \\
\hline & $\mathrm{I}_{3}$ & $8.14 \mathrm{c}$ & $8.26 \mathrm{~d}$ & $14.08 \mathrm{~d}$ \\
\hline \multirow{2}{*}{\multicolumn{2}{|c|}{$\begin{array}{c}\text { Level of significance } \\
\text { CV }(\%)\end{array}$}} & $* *$ & $*$ & $*$ \\
\hline & & 3.91 & 4.49 & 5.23 \\
\hline
\end{tabular}

In a column, means followed by different letter(s) differed significantly by Tukey's test at $\mathrm{P} \leq 5 \%$ level of probability. $\mathrm{F}_{1}$ - Inorganic (control), $\mathrm{F}_{2}$ - Compost $\left(10 \mathrm{tha} \mathrm{h}^{-1}\right)+$ inorganic, $\mathrm{F}_{3}-$ Compost $\left(20 \mathrm{t} \mathrm{ha}^{-1}\right)$ + without inorganic and $\mathrm{I}_{1}$ - continuous flooding, $\mathrm{I}_{2}-100 \%$ field capacity, $\mathrm{I}_{3}-75 \%$ field capacity.

\subsection{Leaf dry weight per plant}

Leaf dry weight per plant was affected by the interaction between fertilizer and different water level (Table 5). At vegetative stage, the highest leaf dry weight per plant $(7.36 \mathrm{~g})$ was obtained in $\mathrm{F}_{2 \times} \mathrm{I}_{1}$ (compost $10 \mathrm{t} \mathrm{ha}^{-1}+$ inorganic with Flooding) which was statistically similar (7.35g) with $\mathrm{F}_{3 \times} \mathrm{I}_{1}$ (compost-20 $\mathrm{t} \mathrm{ha}^{-1}+$ without inorganic with Flooding). The lowest leaf dry weight per plant (4.37 g) was obtained in $\mathrm{F}_{3 \times} \mathrm{I}_{2}$ (compost-20 $\mathrm{t} \mathrm{ha}{ }^{-1}+$ without inorganic) with $(100 \%$ Field capacity).

At booting stage, the highest leaf dry weight per plant $\left(9.65 \mathrm{~g}\right.$ ) was obtained in $\mathrm{F}_{2 \times} \mathrm{I}_{1}$ (compost $10 \mathrm{t}$ $\mathrm{ha}^{-1}+$ inorganic with Flooding) which was statistically similar $(9.85 \mathrm{~g})$ in $\mathrm{F}_{3 \times} \mathrm{I}_{1}$ (compost-20 $\mathrm{t}$ $\mathrm{ha}^{-1}+$ without inorganic with Flooding).The lowest leaf dry weight per plant (4.6 g) was obtained in $\mathrm{F}_{2} \mathrm{I}_{3}$ (compost $10 \mathrm{t} \mathrm{ha}^{-1}+$ inorganic with $75 \%$ Field capacity).

At anthesis stage, the highest leaf dry weight per plant $\left(16.43 \mathrm{~g}\right.$ ) was obtained in $\mathrm{F}_{3 \times} \mathrm{I}_{1}$ (compost $20 \mathrm{t} \mathrm{ha}^{-1}+$ without inorganic with Flooding) which was statistically similar with $F_{2 \times} I_{1}$ (compost $10 \mathrm{t} \mathrm{ha}^{-1}+$ inorganic with Flooding) $(15.39 \mathrm{~g})$. Contrary, the lowest leaf dry weight per plant $(9.59 \mathrm{~g})$ was obtained in $\mathrm{F}_{3} \mathrm{I}_{2}$ (compost-20 t ha ${ }^{-1}+$ without inorganic with $100 \%$ Field capacity).

\subsection{Root dry weight per plant}

Root dry weight per plant was affected by interaction between fertilizer and different water level as presented in Table 6. At vegetative stage, the highest root dry weight per plant (18.6g) was obtained in $\mathrm{F}_{3 \times} \mathrm{I}_{1}$ (compost-20 $\mathrm{t} \mathrm{ha}^{-1}$ + without inorganic with Flooding) which was statistically similar $(15.05 \mathrm{~g})$ to $\mathrm{F}_{1 \times} \mathrm{I}_{1}$ (inorganic with Flooding). The lowest root dry weight per plant $\left(6.72 \mathrm{~g}\right.$ ) was obtained in $\mathrm{F}_{2 \times} \mathrm{I}_{3}$ (compost $10 \mathrm{t}$ $\mathrm{ha}^{-1}+$ inorganic with $75 \%$ Field Capacity) which was statistically similar $(7.90 \mathrm{~g})$ to $\mathrm{F}_{3 \times} \mathrm{I}_{2}$ (compost-20 t ha ${ }^{-1}+$ without inorganic with 100\% Field Capacity).

At booting stage, the highest root dry weight per plant $\left(34.9 \mathrm{~g}\right.$ ) was obtained in $\mathrm{F}_{1 \times} \mathrm{I}_{1}$ (inorganic with Flooding) which was statistically similar with $\mathrm{F}_{1 \times} \mathrm{I}_{3}$ (inorganic with $75 \%$ Field Capacity) $(33.27 \mathrm{~g})$, and the lowest root dry weight per plant (8.26 g) was obtained in $\mathrm{F}_{3 \times} \mathrm{I}_{3}$ (compost-20 $\mathrm{t} \mathrm{ha}^{-1}+$ without inorganic with $75 \%$ field capacity). At anthesis stage, the highest root dry weight per plant $(54.62 \mathrm{~g})$ was obtained in $\mathrm{F}_{1 \times} \mathrm{I}_{1}$ 
(inorganic with Flooding) and the lowest root dry weight per plant $(14.08 \mathrm{~g})$ was obtained in $\mathrm{F}_{3 \times} \mathrm{I}_{3}$ (compost-20 $\mathrm{t} \mathrm{ha}^{-1}+$ without inorganic with $75 \%$ field capacity) which was statistically similar with $\mathrm{F}_{2} \mathrm{I}_{3}$ compost $10 \mathrm{t} \mathrm{ha}^{-1}+$ inorganic with $75 \%$ field capacity) (14.76g). Flooding irrigation with inorganic fertilizer may have increased availability of nutrients that might have produced higher root dry weight as reported similarly by Zubaer et al. (2007).

Table 7. Effect of fertilizer and different water level on leaf area $\left(\mathrm{cm}^{2}\right)$ of rice (BRRI dhan28) at different days after transplanting.

\begin{tabular}{|c|c|c|c|c|c|}
\hline Fertilizer & Irrigation & Vegetative & Booting & Anthesis & Maturity \\
\hline \multirow[t]{3}{*}{$\mathrm{F}_{1}$} & $\mathrm{I}_{1}$ & $1611.93 b$ & $2028.21 \mathrm{a}$ & $4492.47 b$ & $1566.88 b$ \\
\hline & $\mathrm{I}_{2}$ & $1536.4 b$ & $1767.74 b$ & $5286.48 \mathrm{a}$ & $1491.15 b$ \\
\hline & $\mathrm{I}_{3}$ & $2367.05 a$ & $1687.45 b$ & $3968.91 b c$ & $1643.54 \mathrm{a}$ \\
\hline \multirow[t]{3}{*}{$\mathrm{F}_{2}$} & $\mathrm{I}_{1}$ & $1521.53 b$ & $1781.27 \mathrm{~b}$ & $4268.34 b$ & $1507.95 b$ \\
\hline & $\mathrm{I}_{2}$ & $1741.63 b$ & $1664.07 \mathrm{~b}$ & $4627.63 b$ & $1410.14 b c$ \\
\hline & $\mathrm{I}_{3}$ & $1541.33 \mathrm{bc}$ & $1768.76 b$ & $4094.13 b$ & $1289.04 d$ \\
\hline \multirow[t]{3}{*}{$\mathrm{F}_{3}$} & $\mathrm{I}_{1}$ & $1583.83 \mathrm{bc}$ & $1664.78 b$ & $4407.25 b$ & $1287.88 \mathrm{~d}$ \\
\hline & $\mathrm{I}_{2}$ & $1588.9 \mathrm{bc}$ & $1653.85 b c$ & $3978.37 \mathrm{c}$ & $1534.49 b$ \\
\hline & $\mathrm{I}_{3}$ & $1433.86 \mathrm{c}$ & $1572.26 \mathrm{c}$ & $4993.03 \mathrm{ab}$ & $1634.90 \mathrm{a}$ \\
\hline \multicolumn{2}{|c|}{ Level of significance } & $*$ & $* *$ & $*$ & $*$ \\
\hline \multicolumn{2}{|c|}{$\mathrm{CV}(\%)$} & 5.41 & 5.13 & 6.27 & 4.95 \\
\hline
\end{tabular}

In a column, means followed by different letter(s) differed significantly by Tukey's test at $\mathrm{P} \leq 5 \%$ level of probability. $\mathrm{F}_{1}$ - Inorganic (control), $\mathrm{F}_{2}$ - Compost $\left(10 \mathrm{tha}^{-1}\right)+$ inorganic, $\mathrm{F}_{3}-$ Compost $\left(20 \mathrm{t} \mathrm{ha}^{-1}\right)$ + without inorganic and $\mathrm{I}_{1}$ - continuous flooding, $\mathrm{I}_{2}-100 \%$ field capacity, $\mathrm{I}_{3}-75 \%$ field capacity.

Table 8. Effect of fertilizer and water level on some physiological parameters of rice (BRRI dhan28) at anthesis.

\begin{tabular}{|c|c|c|c|c|c|c|}
\hline Fertilizer & Irrigation & $\begin{array}{l}\text { Chlorophyll a } \\
\left(\mathrm{mgg}^{-1}\right)\end{array}$ & $\begin{array}{l}\text { Chlorophyll b } \\
\left(\mathrm{mgg}^{-1}\right)\end{array}$ & $\begin{array}{c}\text { Total } \\
\text { Chlorophyll } \\
\left(\mathrm{mgg}^{-1}\right)\end{array}$ & $\begin{array}{l}\text { Proline } \\
\text { ( } \mu \text { moles } \\
\left.\mathrm{g}^{-1} \mathrm{FW}\right)\end{array}$ & $\begin{array}{c}\text { Relative } \\
\text { Water } \\
\text { Content }(\%) \\
\end{array}$ \\
\hline \multirow[t]{3}{*}{$\mathrm{F}_{1}$} & $\mathrm{I}_{1}$ & $0.9 \mathrm{c}$ & $0.33 b c$ & $0.42 b$ & $0.336 b$ & $21.53 c$ \\
\hline & $\mathrm{I}_{2}$ & $0.13 \mathrm{a}$ & $0.52 \mathrm{a}$ & $0.65 \mathrm{a}$ & $0.332 b c$ & $21.78 \mathrm{c}$ \\
\hline & $\mathrm{I}_{3}$ & $0.04 \mathrm{~d}$ & $0.23 \mathrm{c}$ & $0.27 b c$ & $0.389 \mathrm{a}$ & $22.73 b$ \\
\hline \multirow[t]{3}{*}{$\mathrm{F}_{2}$} & $\mathrm{I}_{1}$ & $0.13 \mathrm{a}$ & $0.41 b$ & $0.54 \mathrm{~b}$ & $0.331 \mathrm{c}$ & $21.52 \mathrm{c}$ \\
\hline & $\mathrm{I}_{2}$ & $0.06 \mathrm{bc}$ & $0.33 b c$ & $0.39 b c$ & $0.333 b$ & $21.38 \mathrm{c}$ \\
\hline & $\mathrm{I}_{3}$ & $0.11 b$ & $0.40 \mathrm{~b}$ & $0.51 \mathrm{~b}$ & $0.371 \mathrm{~b}$ & $23.12 \mathrm{a}$ \\
\hline \multirow[t]{3}{*}{$\mathrm{F}_{3}$} & $\mathrm{I}_{1}$ & $0.04 \mathrm{~d}$ & $0.33 b$ & $0.37 \mathrm{~b}$ & $0.331 \mathrm{c}$ & $20.97 d$ \\
\hline & $\mathrm{I}_{2}$ & $0.13 \mathrm{a}$ & $0.48 \mathrm{ab}$ & $0.62 \mathrm{a}$ & $0.344 b$ & $22.46 b$ \\
\hline & $\mathrm{I}_{3}$ & $0.11 \mathrm{~b}$ & $0.33 b c$ & $0.45 \mathrm{c}$ & $0.347 \mathrm{~b}$ & $22.24 b$ \\
\hline \multicolumn{2}{|c|}{ Level of significance } & $*$ & $*$ & $*$ & $*$ & $*$ \\
\hline \multicolumn{2}{|c|}{$\mathrm{CV}(\%)$} & 5.61 & 3.47 & 4.81 & 3.29 & 7.29 \\
\hline
\end{tabular}

In a column, means followed by different letter(s) differed significantly by Tukey's test at $\mathrm{P} \leq 5 \%$ level of probability. $\mathrm{F}_{1}$ - Inorganic (control), $\mathrm{F}_{2}$ - Compost $\left(10 \mathrm{tha}^{-1}\right)+$ inorganic, $\mathrm{F}_{3}-$ Compost $\left(20 \mathrm{t} \mathrm{ha}^{-1}\right)$ + without inorganic and $\mathrm{I}_{1}$ - continuous flooding, $\mathrm{I}_{2}-100 \%$ field capacity, $\mathrm{I}_{3}-75 \%$ field capacity. 


\subsection{Leaf area}

Leaf area was evaluated for poultry litter based composts in rice production presented in table 7 . Leaf area was found with almost similar in each treatment. Leaf area significantly varied due to different poultry litter based composts. The maximum leaf area were recorded from $F_{2}$ (compost $10 \mathrm{t} \mathrm{ha}^{-1}+$ inorganic) Fertilizer $\left(1622.31,1861.88,4630.83\right.$ and $1878.59 \mathrm{~cm}^{2}$ at vegetative, booting, anthesis and maturity stages, respectively) which was significantly different from other treatments.

Leaf area significantly varied due to different water levels. The maximum leaf area was recorded from $\mathrm{I}_{2}$ (100\% Field Capacity) (1601.5, $1704.70,4530.03$ and $1602.38 \mathrm{~cm}^{2}$ at vegetative, booting, anthesis and maturity stages, respectively) which was significantly different from other treatments.

At vegetative stage, the highest leaf area $\left(2367.05 \mathrm{~cm}^{2}\right)$ was obtained in $\mathrm{F}_{3 \times} \mathrm{I}_{1}$ (compost-20 $\mathrm{t} \mathrm{ha}^{-1}+$ without inorganic with flooding irrigation) and the lowest leaf area $\left(1433.86 \mathrm{~cm}^{2}\right)$ was obtained in $\mathrm{F}_{3 \times} \mathrm{I}_{3}$ (compost-20 $\mathrm{t} \mathrm{ha}^{-1}+$ without inorganic with $75 \%$ Field Capacity).

At booting stage, the highest leaf area (2028.21 $\mathrm{cm}^{2}$ ) was obtained in $\mathrm{F}_{1 \times} \mathrm{I}_{1}$ (inorganic with flooding) and the lowest leaf area $\left(1572.26 \mathrm{~cm}^{2}\right)$ was obtained in $\mathrm{F}_{3 \times} \mathrm{I}_{3}$ (compost-20 $\mathrm{t} \mathrm{ha}^{-1}+$ without inorganic with $75 \%$ field capacity)

At anthesis stage, the highest Leaf area (5286.48 $\mathrm{cm}^{2}$ ) was obtained in $\mathrm{F}_{1 \times} \mathrm{I}_{2}$ (inorganic with $100 \%$ field capacity) which was statistically similar with $\mathrm{F}_{1 \times} \mathrm{I}_{2}$ (inorganic with $100 \%$ field capacity) $\left(4993.03 \mathrm{~cm}^{2}\right)$ and the lowest leaf area $(3978.37$ $\mathrm{cm}^{2}$ ) was obtained in $\mathrm{F}_{3 \times} \mathrm{I}_{3}$ (compost-20 t ha ${ }^{-1}+$ without inorganic with $75 \%$ field capacity).

At maturity stage, the highest leaf area was $\left(1643.54 \mathrm{~cm}^{2}\right.$ ) obtained in $\mathrm{F}_{1 \times} \mathrm{I}_{3}$ (inorganic with $75 \%$ field capacity) which was statistically similar with $\mathrm{F}_{3 \times} \mathrm{I}_{3}$ (compost-20 $\mathrm{t} \mathrm{ha}^{-1}+$ without inorganic with $75 \%$ field capacity) $\left(1634.90 \mathrm{~cm}^{2}\right)$ and the lowest leaf area $\left(1287.88 \mathrm{~cm}^{2}\right)$ was obtained in $\mathrm{F}_{3 \times} \mathrm{I}_{1}$ (compost-20 $\mathrm{t} \mathrm{ha}^{-1}+$ without inorganic with flooding irrigation) which was statistically similar in $\mathrm{F}_{2 \times} \mathrm{I}_{3}$ (compost $10 \mathrm{t} \mathrm{ha}^{-1}+$ inorganic with $75 \%$ field capacity) (1289.04 $\left.\mathrm{cm}^{2}\right)$.

Flooding irrigation with $20 \mathrm{t} \mathrm{ha}^{-1}$ compost may have increased nutrients uptake, which probably helped to produce maximum leaf area as found similar findings by Altieri et al. (2003).

\subsection{Chlorophyll content}

Chlorophyll a content of leaf was significantly increased by the interaction effect of poultry based compost and different water level (Table $8)$. The highest chlorophyll a $\left(0.13 \mathrm{mgg}^{-1}\right)$ was recorded from $\mathrm{F}_{1 \times} \mathrm{I}_{1}$ (inorganic with flooding irrigation) and also $\mathrm{F}_{2 \times} \mathrm{I}_{1}$ (compost ${ }^{-1} 0 \mathrm{t} \mathrm{ha}^{-1}+$ inorganic with flooding irrigation). The lowest chlorophyll a $\left(0.04 \mathrm{mgg}^{-1}\right)$ was recorded from $\mathrm{F}_{1 \times} \mathrm{I}_{3}$ (inorganic with $75 \%$ field capacity) and $\mathrm{F}_{3 \times} \mathrm{I}_{1}$ (compost-20 $\mathrm{t} \mathrm{ha}^{-1}+$ without inorganic fertilizer with flooding irrigation).

Chlorophyll b content of leaf was influenced significantly by the interaction effect of poultry based compost and different water level (Table $8)$. The highest chlorophyll b $\left(0.52 \mathrm{mgg}^{-1}\right)$ was recorded from $\mathrm{F}_{1 \times} \mathrm{I}_{2}$ (inorganic with $100 \%$ field capacity). The lowest chlorophyll b $\left(0.23 \mathrm{mgg}^{-1}\right)$ was recorded from $\mathrm{F}_{1 \times} \mathrm{I}_{3}$ (inorganic with $75 \%$ field capacity).

Total chlorophyll content was influenced significantly by the interaction effect of poultry based compost and different water level (Table $8)$. The highest total chlorophyll $\left(0.65 \mathrm{mgg}^{-1}\right)$ was observed in $\mathrm{F}_{1 \times} \mathrm{I}_{2}$ (inorganic with $100 \%$ field capacity) which was statistically similar in $\mathrm{F}_{3 \times} \mathrm{I}_{2}$ (compost-20 t ha ${ }^{-1}+$ without inorganic with $100 \%$ field capacity).The lowest total chlorophyll $\left(0.27 \mathrm{mgg}^{-1}\right)$ was recorded from $\mathrm{F}_{1 \times} \mathrm{I}_{3}$ (inorganic with $75 \%$ field capacity). Zubaer et al. (2007) and Sokoto and Muhammad (2014) found the similar result at drought condition that compost and nutrients increased chlorophyll contents in crop plant. 


\subsection{Proline content}

The interaction of poultry based compost and different water level influenced proline content of leaf significantly at anthesis stage (Table 8). The highest proline content $\left(0.389 \mu\right.$ moles $\mathrm{g}^{-1}$ FW) was recorded from $\mathrm{F}_{1 \times} \mathrm{I}_{3}$ (inorganic fertilizer with $75 \%$ field capacity). The lowest proline content $\left(0.331 \mu\right.$ moles $\left.\mathrm{g}^{-1} \mathrm{FW}\right)$ was recorded from $F_{2 \times} I_{1}$ (compost $10 \mathrm{t} \mathrm{ha}^{-1}+$ inorganic with flooding irrigation) and which was statistically similar with $\mathrm{F}_{3 \times} \mathrm{I}_{1}$ (compost-20 $\mathrm{t} \mathrm{ha}^{-1}+$ without inorganic with flooding irrigation). The proline content increases due to water stress situation but the application of higher compost may decrease proline content. This might be occurred due to .increase water holding capacity of compost (Zubaer et al., 2007).

\subsection{Relative leaf water content}

The interaction of poultry based composts and different water level also influenced the relative water content of leaf significantly (Table 8). The highest relative leaf water content (23.12) was recorded from $\mathrm{F}_{2 \times} \mathrm{I}_{3}$ (compost $10 \mathrm{t} \mathrm{ha}^{-1}+$ inorganic fertilizer with $75 \%$ field capacity). The lowest relative leaf water content (20.97) was observed in $\mathrm{F}_{3 \times} \mathrm{I}_{1}$ (compost-20 $\mathrm{t} \mathrm{ha}^{-1}+$ without inorganic with flooding).

\subsection{Panicle length}

Interaction between fertilizer and different water level significantly influenced the panicle length (Table 9). The longest panicle (23.12) found in $\mathrm{F}_{2 \times} \mathrm{I}_{3}$ (compost $-10 \mathrm{t} \mathrm{ha}^{-1}+$ inorganic with $75 \%$ field capacity) treatment and the shortest one (20.97) found in $F_{3 \times} I_{1}$ (compost $\left(20 \mathrm{t} \mathrm{ha}^{-1}\right)+$ without inorganic with flooding) treatment. Integration of compost and inorganic fertilizers may provide available nutrients for longer time with flooding irrigation. Sarker et al. (2013) and Ali et al. (2009) found similar results of increased panicle length due to application of manure on rice plant.

\subsection{0-grains weight}

Interaction effect of fertilizer and different water level significantly influenced the 1000-grain weight (table 9). The highest 1000-grain weight $(19.13 \mathrm{~g})$ was recorded from $\mathrm{F}_{3 \times} \mathrm{I}_{3}$ treatment (compost-20 t ha ${ }^{-1}+$ without inorganic with $75 \%$ field capacity) which was statistically similar with $\mathrm{F}_{1 \times} \mathrm{I}_{3}$ and $\mathrm{F}_{2 \times} \mathrm{I}_{3}$ treatments. The lowest 1000 grain weight $(16.17 \mathrm{~g})$ was recorded from $\mathrm{F}_{3 \times} \mathrm{I}_{1}$ treatment (compost $-20 \mathrm{t} \mathrm{ha}^{-1}+$ without inorganic with $75 \%$ field capacity).

Table 9. Effect of fertilizer and water level on yield parameters of rice (BRRI dhan28) at harvest.

\begin{tabular}{|c|c|c|c|c|c|c|}
\hline Fertilizer & Irrigation & $\begin{array}{c}\text { Panicle } \\
\text { length }(\mathrm{cm})\end{array}$ & $\begin{array}{l}\text { 1000-grain } \\
\text { weight }(\mathrm{g})\end{array}$ & $\begin{array}{c}\text { Grain } \\
\text { yield/ pot }(\mathrm{g})\end{array}$ & $\begin{array}{c}\text { Straw yield } \\
(\mathrm{g})\end{array}$ & $\begin{array}{c}\text { Harvest } \\
\text { index }(\%)\end{array}$ \\
\hline \multirow[t]{3}{*}{$\mathrm{F}_{1}$} & $\mathrm{I}_{1}$ & $21.53 \mathrm{~b}$ & $17.97 \mathrm{~b}$ & $28.44 \mathrm{~b}$ & $30.47 \mathrm{~b}$ & $48.27 b$ \\
\hline & $\mathrm{I}_{2}$ & $21.78 b$ & $17.8 b$ & $28.95 b$ & $33.68 \mathrm{a}$ & $46.22 b c$ \\
\hline & $\mathrm{I}_{3}$ & $22.73 a$ & $18.97 \mathrm{a}$ & $25.83 \mathrm{c}$ & $24.77 b c$ & $51.04 \mathrm{a}$ \\
\hline \multirow[t]{3}{*}{$\mathrm{F}_{2}$} & $\mathrm{I}_{1}$ & $21.52 b$ & $17.7 b$ & $32.30 \mathrm{a}$ & $35.97 \mathrm{a}$ & $47.32 b$ \\
\hline & $\mathrm{I}_{2}$ & $21.38 \mathrm{~b}$ & $18.83 \mathrm{a}$ & $19.20 \mathrm{c}$ & $26.28 b$ & $42.21 \mathrm{~d}$ \\
\hline & $\mathrm{I}_{3}$ & $23.12 \mathrm{a}$ & $17.8 \mathrm{~b}$ & $16.9 \mathrm{~d}$ & $20 \mathrm{c}$ & $45.79 \mathrm{c}$ \\
\hline \multirow[t]{3}{*}{$\mathrm{F}_{3}$} & $\mathrm{I}_{1}$ & $20.97 \mathrm{c}$ & $17.17 b$ & $33.17 \mathrm{a}$ & $31.17 \mathrm{ab}$ & $51.55 \mathrm{a}$ \\
\hline & $\mathrm{I}_{2}$ & $22.46 b$ & $17.77 b$ & $20.90 b c$ & $27.35 b$ & $43.31 \mathrm{c}$ \\
\hline & $\mathrm{I}_{3}$ & $22.24 b$ & $19.13 \mathrm{a}$ & $18.08 \mathrm{c}$ & $19.03 \mathrm{c}$ & $48.72 b$ \\
\hline \multicolumn{2}{|c|}{ Level of significance } & $* *$ & $*$ & $*$ & $* *$ & $*$ \\
\hline \multicolumn{2}{|c|}{$\mathrm{CV}(\%)$} & 8.31 & 5.31 & 4.87 & 4.81 & 6.31 \\
\hline
\end{tabular}

In a column, means followed by different letter(s) differed significantly by Tukey's test at $\mathrm{P} \leq 5 \%$ level of probability. $\mathrm{F}_{1}$ - Inorganic (control), $\mathrm{F}_{2}$ - Compost $\left(10 \mathrm{tha} \mathrm{ha}^{-1}\right)+$ inorganic, $\mathrm{F}_{3}-$ Compost $\left(20 \mathrm{t} \mathrm{ha}^{-1}\right)+$ without inorganic and $\mathrm{I}_{1}$ - continuous flooding, $\mathrm{I}_{2}-100 \%$ field capacity and $\mathrm{I}_{3}-75 \%$ field capacity. 


\subsection{Grain yield}

Interaction effect of fertilizer and different levels of different water level was significant influenced in grain yield (Table 9). The highest grain yield (33.17 $\left.\mathrm{g} \mathrm{pot}^{-1}\right)$ was recorded from $\left(\mathrm{F}_{3 \times} \mathrm{I}_{1}\right)$ (compost $\left(20 \mathrm{t} \mathrm{ha}^{-1}\right)+$ without inorganic) with flooding. The lowest grain yield (16.9 $\mathrm{g} \mathrm{pot}^{-}$ $\left.{ }^{1}\right)$ was recorded from $\left(\mathrm{F}_{2 \times} \mathrm{I}_{3}\right)$ (compost $\left(10 \mathrm{t} \mathrm{ha}^{-1}\right)$ + inorganic) with (75\% field capacity). Water stress $\left(\mathrm{I}_{3}\right)$ may be caused lowest grain yield due less food partioning whereas flood irrigation $\left(\mathrm{I}_{1}\right)$ produce the higher grain yield per pot. Rahman et al. (2018), Tilahun et al. (2013) and Saleque et al. (2004) reported that application of compost with inorganic fertilizer increased yield of rice.

\subsection{Straw Yield}

Combined effect of compost and different water levels significantly influenced the straw yield (Table 9). The highest straw yield (35.97 g) was recorded from $\mathrm{F}_{2 \times} \mathrm{I}_{1}$ (compost $10 \mathrm{t} \mathrm{ha}^{-1}+$ inorganic with flooding irrigation). The lowest straw yield $(19.03 \mathrm{~g})$ was recorded from $\mathrm{F}_{3 \times} \mathrm{I}_{3}$ (compost-20 t ha ${ }^{-1}+$ without inorganic with $75 \%$ field capacity) treatment. Tilahun et al. (2013), Hossain et al. (2010) and Man et al. (2007) also reported that compost and irrigation together increased biomass yield in field crop.

\subsection{Harvest index (\%)}

Combined effect of poultry litter based compost and different water levels significantly influenced harvest index (Table 9). The highest harvest index $(51.55 \%)$ was recorded from compost-20 t ha ${ }^{-1}+$ without inorganic fertilizer with flooding $\left(\mathrm{F}_{3 \times} \mathrm{I}_{1}\right)$ irrigation which was statistically similar with $\mathrm{F}_{1 \times} \mathrm{I}_{3}$ (inorganic with $75 \%$ field capacity). The lowest harvest index $(42.21 \%)$ was recorded from compost $10 \mathrm{t} \mathrm{ha}^{-1}+$ inorganic with $100 \%$ field capacity $\left(\mathrm{F}_{2 \times} \mathrm{I}_{2}\right)$. Due to increase of grain yield harvest index increased. Islam et al. (2014), Tilahun et al. (2013) and Man et al. (2007) also reported the similar result that application of nutrients and irrigation increased harvest index in cereal.

\section{Conclusions}

The influence of organic fertilizer application on crop production and soil properties can be achieved after long time applications. However from the present study, one year poultry litter based compost addition increased the production of BRRI Dhan28. The combined application of poultry litter based compost and inorganic fertilizers increased the rice production and yield through enhanced tiller number, panicle length, 1000-grain weight presumably by increasing nutrient uptake and utilization. From the point of view of sustainable production, integrated application of $10 \mathrm{t}^{-1}$ ha ${ }^{-1}$ poultry litter based compost with inorganic fertilizer is recommended for rice cultivation in this area.

\section{Acknowledgement}

This research work was funded by IRT, Hajee Mohammad Danesh Science and Technology University, Dinajpur-5200.

\section{References}

Abro MB., Abbasi ZA., Maitlo SA., Maitlo ND., Bhatti AG., Panhwar NA. 2002. Growth response of rice variety $\mathrm{DR}-82$ as influenced by green manure (Dhaincha). Pakistan Journal of Applied Science, 27 (7):781-782.

Adugna G. 2016. A review on impact of compost on soil properties, water use and crop productivity. Academic Research Journal of Agricultural Science and Research, 4(3): 93 -104.

AIS (Agriculture Information Service). 2008. Krishi Diary. Agric. info. Service, Khamarbari, Dhaka.66p.

Akter, S. 2011. Effects of nitrogen supplied from manures and fertilizers on the growth and yield of BRRI dhan29. MS thesis, Department of Soil Science, Bangladesh Agricultural University, Mymensingh 2202. 
Ali ME., Islam MR., Jahiruddin M. 2009. Effect of integrated use of organic manure with chemical fertilizers in the rice-rice cropping system and its impact on soil health. Bangladesh Journal of Agricultural. Research, 34(1): 81-90.

Altieri M A., Nicholls CI. 2003. Soil fertility management and insect pests: harmonizing soil and plant health in agroecosystems. Soil and Tillage Research, 72(2):203-211.

Barrs SD., Weatherley PE. 1962. Are examination of relative turgidity technique for estimating water deficits in leaves. Australian Journal of biological Science, 15: 413-428.

BBS (Bangladesh Bureau of Statistics). 2014. Statistical Yearbook of Bangladesh. Statistics Division, Ministry of Planning, Govt. of Peoples Republic of Bangladesh, Dhaka.

Bair W. 1990. Characterization of the environment for sustainable agriculture in Semi-arid Tropics. In: Proc. Sustainable Agriculture Issue, Prospective and Prospects in Semi-arid Tropics (ed. Singh, R.P.), Hydderabad, India. India Society of Agronomy, 90-128pp.

Chowdhury AKMMB., Akratos CS., Vayenas DV., Pavlou S. 2013. Olive mill waste composting: a review. International Journal of Biodeterioration \& Biodegradation, 85: 108-119.

Diacono M., Montemurro F.2010. Long-term effects of organic amendments on soil fertility-A review. Agronomy and. Sustainable Development, 30: 401-422.

Islam F., Khan MA., Bari ASMF., Hosain MT., Sabikunnaher. 2013: Effect of Fertilizer and Manure on the Growth, Yield and Grain Nutrient Concentration of Boro Rice (Oryza sativa L.) under Different Water Management Practices. The Agriculturists. 11 (2): 44-51.
Haque A. 2013. Integrated use of manures and fertilizers for maximizing the growth and yield of Boro rice (cv. BRRI dhan28). MS Thesis, Department of Soil Science, Bangladesh Agricultural University, Mymensingh 2202.

Hasan MN. 2014. Effect of usg with cowdung on nitrogen use efficiency of rice and nitrogen dynamics in porewater. $M S$ Thesis, Department of Soil Science, Bangladesh Agricultural University, Mymensingh 2202.

Hoshain S. 2010. Effect of cowdung and nitrogen (urea) on growth, yield and yield contributing characters of aromatic rice cv. BRRI dhan50. MS Thesis. Department of Agronomy, Bangladesh Agricultural University, Mymensingh 2202.

Hossen MA., Shamsuddoha ATM., Paul AK., Bhuiyan MSI., Zobaer ASM. 2011. Efficacy of different organic manures and inorganic fertilizer on the yield and yield attributes of Boro rice. The Agriculturists, 9 (1\&2): 117-125.

Hossain MI., Uddin MN., Islam MS., Hossain MK. Khan MAH. 2010. Effects of manures and fertilizer on nutrient content and uptake by BRRI dhan29. Journal of Agroforestry and Environment, 3(2): 6567.

Islam MR., Rashid MB., Siddique AB., Afroz H. 2014. Integrated effects manures and fertilizers on the yield and nutrient uptake by BRRI dhan49. Journal of Bangladesh Agricultural University, 12(1): 67-72.

Kobra UEZ. 2016. Valorization of Poultry Wastes by Aerobic Composting for the Organic Agriculture. MS Thesis. Department of Crop Physiology and Ecology, Hajee Mohammad Danesh Science and Technology University, Dinajpur 5200.

Lin X., Zhu D., Lin X. 2011. Effects of water management and organic fertilization with SRI crop practices on hybrid rice performance and rhizosphere dynamics. 
Paddy and Water Environment, 9(1), 3339.

Liu CA., Li FR., Zhou LM., Zhang RH., Jia Y., Lin SL., Wang LJ., Siddique KHM., Li FM.2013. Effect of organic manure and fertilizer on soil water and crop yields in newly-built terraces with loess soils in a semi-arid environment. Agricultural Water Management, 117:123-132.

Man LH., Khang VT., Watanabe T. 2007. Improvement of soil fertility by rice straw manure. Omon Rice (15), 124 134.

Marinari S., Masciandro G., Ceccanti B., Grego S. 2000. Influence of organic and mineral fertilisers on soil biological and physical properties. Bioresource Technology, 72(1):9-17.

Meelu OP., Singh Y. 1991. Integrated use of fertilizers and organic manure for higher returns. Progressive Farming Punjab Agricultural University, 27: 3-4.

Najafi N., Abbasi M. 2013. Effects of soil water conditions, sewage sludge, poultry manure and chemical fertilizers on macronutrients concentrations in rice plant. International Journal of Agronomy and Plant Production, 4(5): 1066-1077.

Nambiar KKM. 1991. Long-term fertility effects on wheat productivity. In wheat for the Non-traditional Warm areas, (ed.) Saunders D.A., CIMMYT. 516-521

Rahman F. Hossain ATMS., Islam, MR. 2018. Integrated Effects of Poultry Manure and Chemical Fertilizer on Yield, Nutrient Balance and Economics of Wetland Rice Culture. Bangladesh Rice Journal, 22(2):71-77.

Reardon C., Wuest SB. 2016. Soil amendments yield persisting effects on the microbial communities: A 7-year study. Applied Soil Ecology. 101: 107-116.

Saleque MA., Abedin MS., Bhuiyan NI., Zaman SK., Panaullah GM. 2004. Long term effect of inorganic and organic fertilizer sources on yield and nutrient accumulation of lowland rice. Field Crops Research, 86: 53-65.

Shaha U. 2014. Integrated use of cowdung and inorganic fertilizer on the performance of modern varieties of transplanted aman rice. MS Thesis, Department of Agronomy, Bangladesh Agricultural University, Mymensingh, 2202.

Shelley IJ., Takahashi-Nosaka M, Kano-Nakata M., Haque MS., Inukai Y. 2016 .Rice Cultivation in Bangladesh: Present Scenario, Problems, and Prospects. Journal of International Cooperation for Agricultural Development, 14:20-29.

Sokoto MB., Muhammad A. 2014. Response of rice varieties to water stress in Sokoto, Sudan Savannah, Nigeria. Journal of Biosciences and Medicines, 2: 68-74.

Sarker BC., Zahan M., Majumder UK., Islam MA. Roy B. 2013. Growth and yield potential of some local and high yielding Boro rice cultivars. Journal of Agroforestry and Environment, 7(1): 107110.

Tilahun T., Nigussie D., Wondimu, B., Setegn G. 2013. Effect of farmyard manure and inorganic fertilizers on the growth, yield and moisture stress tolerance of rain-fed lowland rice. American Journal of Research Communication, 1(4):275-301.

Urra J., Alkorta I., Garbisu C. 2019. Potential Benefits and Risks for Soil Health Derived From the Use of Organic Amendments in Agriculture. Agronomy, 9 (9): 542-552.

Witham FH., Blaydes DF., Devlin RM.. 1986. Chlorophyll absorption spectrum and quantitative determination. Excercises in plant physiology, $128-131$.

Zubaer MA., Chowdhury AKMMB., Islam MZ., Ahmed T., Hasan MA. 2007. Effects of water stress on growth and yield attributes of aman rice genotypes. International Journal of Sustainable Crop Production, 2(6):25-30. 